\title{
Lugné-Poe et son approche de Shakespeare : de Pompée à Polonius
}

Isabelle Schwartz-Gastine

\section{(2) OpenEdition}

Journals

Édition électronique

URL : http://journals.openedition.org/shakespeare/622

DOI : $10.4000 /$ shakespeare.622

ISSN : 2271-6424

Éditeur

Société Française Shakespeare

Édition imprimée

Date de publication : 1 novembre 2000

Pagination : 159-172

ISBN : 2-84269-407-4

\section{Référence électronique}

Isabelle Schwartz-Gastine, "Lugné-Poe et son approche de Shakespeare : de Pompée à Polonius »,

Actes des congrès de la Société française Shakespeare [En ligne], 18 | 2000, mis en ligne le 01 novembre 2007, consulté le 24 avril 2019. URL : http://journals.openedition.org/shakespeare/622 ; DOI :

$10.4000 /$ shakespeare.622 


\section{Le renouveau élisabéthain}

Lugné-Poe aborde le théâtre élisabéthain par des ouvres moins connues que celles de Shakespeare, par le truchement d'érudits anglais et français tels que Marcel Schwob ; celui-ci lui a signalé le drame de John Ford, Annabella, qu'il portera sur la scène du Théâtre de l'CEuvre, au cours de la deuxième saison, c'est-à-dire en 1894-95, dans une traduction de Maeterlinck. Selon la pratique chère à Lugné-Poe, la représentation est précédée d'une conférence de Marcel Schwob, justement. (Certains, dont Maeterlinck, se sont toujours «défilés» devant cet exercice, qui n'était pas toujours très bien suivi, ou devenait prétexte à chahut, comme on l'a signalé pour Alfred Jarry !). Le drame d'un «post-élisabéthain», Thomas Otway, Venise sauvée, est donné la saison suivante, en 1895, au Théâtre de la Comédie Parisienne, dans une traduction de Gyl Pène, apparemment l'un de ses pseudonymes ${ }^{20}$.

Mais surtout, il s'initie à Shakespeare au cours de ses fréquents voyages à Londres (ses parents y résidaient alors), où il découvre les mises en scène de l'Elizabethan Stage Society, fondée par William Poel et Arthur Dillon, quelque dix ans auparavant ${ }^{21}$. Contrairement à son approche du théâtre scandinave, qu'il traite selon un angle bien personnel comme je viens de le mentionner, Lugné-Poe se penche très attentivement sur les principes de fonctionnement du thêâtre shakespearien. Cependant, il aborde le dramaturge, non pas par la fréquentation du texte comme pour le reste de son parcours, mais par le biais de la pratique théâtrale contemporaine, et ce, dans un souci de reconstitution historique et de fidélité absolue à l'auteur. On peut évidemment douter, avec le recul du temps et d'après les mémoires de Robert Speaight, l'un des grands acteurs impliqués dans l'Elizabethan Stage Society, de la légitimité absolue d'une telle appellation ${ }^{22}$. Mais dans un article intitulé «Shakespeare sans décors» ${ }^{23}$, Lugné-Poe commente la mise en scène des Deux Gentilshommes de Vérone qu'il a vue en $1896^{24}$, ce qui lui permet de dégager certaines thématiques qu'il illustrera ensuite dans sa mise en scène.

La fidélité aux origines de ce théâtre passe par une reconstitution du lieu qui puisse être support d'un style de jeu très particulier. "Jouer les anciens maîtres anglais tels qu'ils furent représentés autrefois à leur époque, tel était donc le but poursuivi par la Elizabethan Stage Society. Mais avant tout début cette... intégrité réclamait un théâtre spécial, et c'est alors que se 
disloquèrent toutes nos théories sur la construction du théâtre à la mode, car aucun ne réunissait les qualités de propriété» ${ }^{25}$. LugnéPoe s'enflamme, avec un certain esprit critique cependant, pour l'utilisation de salles chargées de souvenirs shakespeariens ${ }^{26}$ qui avaient alors une tout autre vocation (comme The Merchant Taylors' Hall pour la représentation dont il est question) ${ }^{27}$ et qui offraient un espace pouvant s'accorder aux principes du théâtre élisabéthain, en particulier la disposition des spectateurs, jouxtant l'espace scénique et répartis sur trois côtés. Le praticable érigé pour la circonstance est inspiré par le croquis du Fortune Theatre («... moins coûteux que celui du Globe», écrit Lugné-Poe avec son esprit pratique qui trahit son manque d'argent chronique).

Il apprécie, entre autres innovations, l'absence de ces lourds rideaux qui tombaient sur la scène parisienne de son époque en interrompant le cours du spectacle. Il a très bien décrit un principe de jeu qu'il a ensuite utilisé, à savoir une continuité d'action située en divers lieux scéniques, avec impossibilité pour les spectateurs de tout suivre visuellement: "[Valentin] doit partir, mais ne croyez pas, comme nous l'avons vu jusqu'alors dans la pièce, qu'il sortira de scène par une des deux baies de droite ou de gauche. Bien mieux, tout affligé, désolé, il s'en va au milieu du public, continuant de jouer son rôle, et, à peine est-il sorti par le fond (public), que nous l'entendons dans une antichambre attaqué par des outlaws. De cette sorte plusieurs des scènes extérieures, si fréquentes dans Shakespeare, se passaient derrière nous et nous entendions tout, sans que l'action cessât une minute sur la scène» ${ }^{28}$.

Retour au texte authentique avec dégraissage des ajouts successifs ${ }^{29}$, costumes somptueux vus «à travers les peintres contemporains de Shakespeare», instruments de musique historiques, les principes de l'Elizabethan Stage Society s'appuyaient sur les recherches de l'époque, et se portaient garant d'authenticité absolue.

Outre ce recentrage sur la véracité historique, Lugné-Poe voyait dans cette démarche l'application de certaines de ses idées, en particulier celles concernant l'absence de décor et la simplicité du geste théâtral. Cette forme de théâtre étranger l'attirait également, car elle lui semblait porteuse de nouvelles expériences théâtrales et esthétiques, puisque son but avoué, qu'il rapporte dans ses mémoires, avec la distance de quelques décennies, était de «révolutionner le plateau» ${ }^{30}$. 
Il prévoyait se lancer dans un «cycle shakespearien» ${ }^{31}$, comme il l'avait fait pour le théâtre scandinave, mais cette tentative tourna court. De cet imposant projet, il ne propose qu'une unique représentation de Mesure pour mesure, pour inaugurer, avec retard, eu égard à des difficultés financières renouvelées, la sixième saison de l'Euvre.

\section{Mesure pour mesure, 10 décembre 1898}

Lugné-Poe investit un espace bien particulier, non balisé pour l'activité théâtrale, le Cirque d'Été, situé sur les Champs Élysées, mis gracieusement à sa disposition par les Concerts Lamoureux, qui, eux, s'y produisaient l'été.

Dans cet espace gigantesque dénué d'habitudes théâtrales, il pouvait retrouver la forme circulaire de l'édifice shakespearien, non pas «the wooden O», mais une construction métallique aux vastes proportions, parcourue de gradins circulaires, offrant des possibilités d'entrées et de sorties de toutes parts. Au milieu de la piste jonchée de sciure, il a planté une charpente de bois montée pour la circonstance, qui délimite le lieu théâtral par ses tréteaux éphémères ouverts sur trois côtés ${ }^{32}$. Le Petit Bleu affirme, sentencieux : «Cette charpente, d'apparence assez rustre, a été érigée sur les indications de la Société Élisabéthaine de Londres; elle est donc d'une absolue rigueur historique».

Le décor de Robert Hista a été établi selon le croquis du Fortune Theatre (voir illustration 1), avec un rideau central flanqué d'une ouverture de part et d'autre. Malheureusement ce dessin ne reproduit pas le balcon blanc, situé au-dessus, qui s'achève par un auvent, décrit par certains journaux. Le proscenium est un espace vide, assurant la proximité des acteurs avec la salle ${ }^{33}$, d'autant plus que quatre marches permettent un accès direct à l'allée centrale. Selon le principe de fonctionnement qu'il a tant admiré à Londres, il fait entrer et sortir les acteurs par la salle, permettant ainsi une continuité du spectacle, des aires de jeu différenciées et mobiles audelà du cadre scénique, et une interaction avec les spectateurs. «On espéra bien révolutionner Paris par nos scènes dans la salle - les premières que l'on vît de la sorte ...», indique Lugné-Poe dans ses mémoires. Cette innovation, en effet, a été remarquée, soulignée par la critique, mais a reçu un écho très divers, certains étant prêts à accepter un principe innovant, d'autres n'ayant pas vraiment compris l'intérêt d'une telle nouveauté. Le commentaire du Petit Bleu est éloquent : «On était légèrement dérouté par ces entrées et 
ces sorties imprévues de personnages qui venaient débiter leurs scènes comme une série de numéros d'un spectacle forain, sans parvenir à découvrir exactement le lien qui unissait ces diverses conversations...».

Contrairement aux indications de l'Elizabethan Stage Society, les costumes semblent avoir été dessinés avec une certaine intemporalité empreinte de sobriété. Lui-même interprète Pompée, sous le pseudonyme de Philippon, dont il donne une interprétation comique et vulgaire qui émoustille le bon goût avec entrain. À propos d'objet scénique, Lugné-Poe mentionne avec émotion dans ses mémoires «le fameux crochet» qui procède d'une recherche de véracité historique que l'on peut qualifier à présent de douteuse, par rapport à l'impact probable sur les spectateurs : «Mais oui, la Société Élisabéthaine me l'avait envoyé : le crochet dont on usait à l'égard des personnes indignes. Le Prévost non plus que le premier bourreau ne devaient porter les mains sur le personnage louche, Pompée "fessier" dont le programme indiquait ainsi clairement la profession. Ce fut une fois qu'il fut arrêté avec un crochet de métal souple en forme de lyre, fixé au bout d'un bâton, qu'on le mena vers son destin, tenu par la gorge» ${ }^{34}$.

On a abondamment parlé du texte de Louis Ménard ${ }^{35}$, traduction, ou plutôt adaptation en alexandrins très contraints (il n'en reste que quatre actes sur cinq). Lugné-Poe aurait été obligé d'accepter ce texte car Ménard aurait subvenu à une bonne partie des frais occasionnés par l'érection du praticable. Mais il paraît aussi qu'il ne s'est pas privé de remanier copieusement le texte pour la représentation. On peut être surpris de la description faite par Lugné-Poe dans ses mémoires, rédigées d'une plume alerte mais rétrospective, d'éléments absolument pas shakespeariens qui n'apparaissent pas dans les commentaires des comptes-rendus, à savoir «la fuite de la fille du duc, les gardes la poursuivant, une scène dans les corridors de la salle avec des bandits, enfin la rentrée par le public du duc ramenant sa fille» ${ }^{36}$. Ces péripéties étaientelles dans le texte finalement porté à la scène ? Lugné-Poe a-t-il un défaut de mémoire, ou bien a-t-il rajouté des éléments qui favorisaient le jeu dans la salle ? Il est difficile d'élucider ce problème complètement par manque de preuves tangibles.

Un critique a qualifié cette mise en scène de jeunesse de «divertissement archéologique» ${ }^{37}$, ce qui est sûr, c'est que LugnéPoe voulait expérimenter une forme théâtrale qui lui semblait nouvelle en France, avec pour but ultime clairement exprimé de 
surprendre son auditoire, cette fois-ci, non pas par une excentricité appuyée comme $U b u$ Roi, mais par un retour à une tradition étrangère qu'il affirme suivre à la lettre n'appartenant pas au registre français habituel. Quelque temps après, il est obligé de fermer l'Euvre pour la première fois. En portant un regard critique sur sa mise en scène, il conclut que «ce curieux spectacle vint au moins vingt ans trop tôt». Il en était de même pour $U b u$ Roi qu'il a repris beaucoup plus tard, en 1922, mais sans aucun scandale. En revanche, sa seconde tentative shakespearienne, bien longtemps après, eut une tout autre réception.

\section{Une deuxième expérience : Hamlet, 1913}

$\mathrm{Au}$ moment où Lugné-Poe s'embarque dans sa deuxième mise en scène shakespearienne, il a dépassé la quarantaine, sa carrière théâtrale est bien établie, faite de scandales, d'innovations, de déboires amers également. Il est très connu à l'étranger, des pays scandinaves à l'Amérique du Sud, il parcourt avidement le monde, comme «ambassadeur» du théâtre français, et il est officiellement remercié à ce titre. Il a mis en scène de très grandes actrices de son temps : la Duse, Isadora Duncan, et bien sûr Suzanne Després, qui fait une carrière remarquable. Mais le public parisien et surtout la critique française l'ont passablement violenté. Cependant, au cours de la dix-huitième saison de l'CEuvre, il retrouve le succès grâce à la mise en scène triomphale de L'Annonce faite à Marie, de Paul Claudel, dans des décors de Jean Variot, selon le principe qu'il a pratiqué depuis longtemps déjà de la synthèse décorative. Le public est prêt à retrouver le chemin de l'Euvre avec intérêt.

Les représentations de Hamlet, dans «une traduction de Georges Duval, couronnée par l'Académie Française, entièrement conforme au texte anglais», sont données du 1er au 16 octobre 1913 au Théâtre Antoine, alors administré par Firmin Gémier. Ironie du sort, on se souvient que Lugné-Poe avait débuté sa carrière d'acteur justement chez Antoine! Gémier interprète le premier fossoyeur, dans les premières représentations uniquement, semble-t-il, à la fois hilare et désenchanté. Lugné-Poe est un Polonius très remarqué car il compose son personnage avec une grande bonhomie qui sait dissimuler son rôle d'espion permanent. Hamlet est pris en charge par Suzanne Després.

Les premiers articles de presse paraissent dès le mois de juin, (pour le ler octobre) faisant état de compilations très érudites, sur le théâtre shakespearien et ses variations de perception, sur les 
changements d'interprétation du personnage d'Hamlet, sur les mises en scène célèbres du passé : c'est un intense débat avec réponses, critiques, dialogue et oppositions, ou précisions. C'est aussi une véritable campagne de publicité organisée autour de ce spectacle qui attise la curiosité de futurs spectateurs. Des photos de Suzanne Després la représentent en train de ferrailler; en effet, elle travaille activement son rôle, prend des cours d'escrime, suit une cure d'amaigrissement, rend visite au metteur en scène Gordon Graig qui avait monté Hamlet au Théâtre d'Art de Moscou, recherche la justesse du personnage. Il s'agit d'un travail en profondeur, tous les critiques insistent sur le fait qu'elle a une approche très personnelle de la compréhension de la pièce et du personnage. Il est également intéressant de constater que c'est elle qui semble être responsable de l'interprétation de son rôle ; elle parât avoir une grande latitude créatrice, ce qui tend à montrer que le rôle du metteur en scène est limité, selon la conception de Lugné-Poe à laquelle on a déjà fait allusion.

Les principes de mises en scène sont plus traditionnels que pour Mesure pour mesure: le théâtre Antoine offre une scène frontale à l'italienne de taille réduite. Le fond de scène est parcouru par un décor unique, fixe : une arche médiévale donnant l'illusion de la profondeur, flanquée de deux ouvertures, mais qui peut évoquer, d'une façon très stylisée, le dispositif de fond de scène élisabéthain. C'est un superbe arrondi, qui indique un lieu clos, immuable, sombre et qui surplombe les personnages de façon imposante lors des nombreux dispositifs en alignement statique tout au fond de l'arche (voir illustration 2).

Plus de toiles bigarrées aux couleurs criardes des premières créations, mais le décor et les costumes d'un autre «jeune» talent de cette époque, Jean Vignot, écrivain, qui venait de se distinguer pour sa participation à L'Annonce faite à Marie. Le principe des toiles peintes est cependant maintenu, mais très sobres dans leur stylisation qui évoque la simplicité médiévale : une toile claire alterne avec une toile foncée, toutes deux représentant des blasons animaliers auxquels répondent les motifs de certains costumes. Il y a deux autres toiles peintes, un extérieur pour le fantôme (que je n'ai pas trouvé), et l'autre pour le cimetière avec des ossements dessinés à gros traits en premier plan et une perspective de croix et de cyprès qui semblent s'étendre à l'infini sur une terre désespérément plate (illustration 3). 


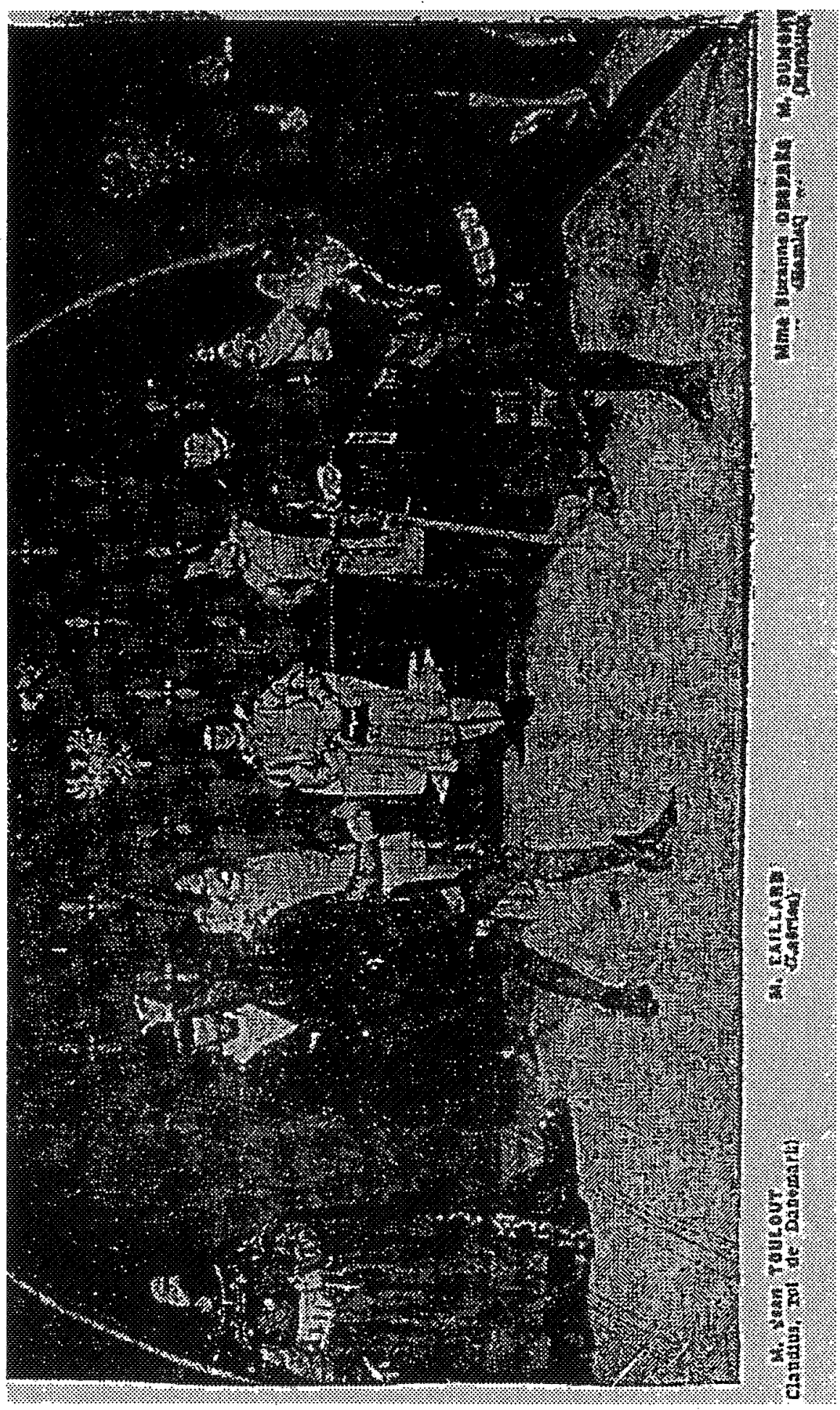

Illustration 5

Hamlet, Théâtre Antoine, 1913

Le duel entre Laertes et Hamlet 


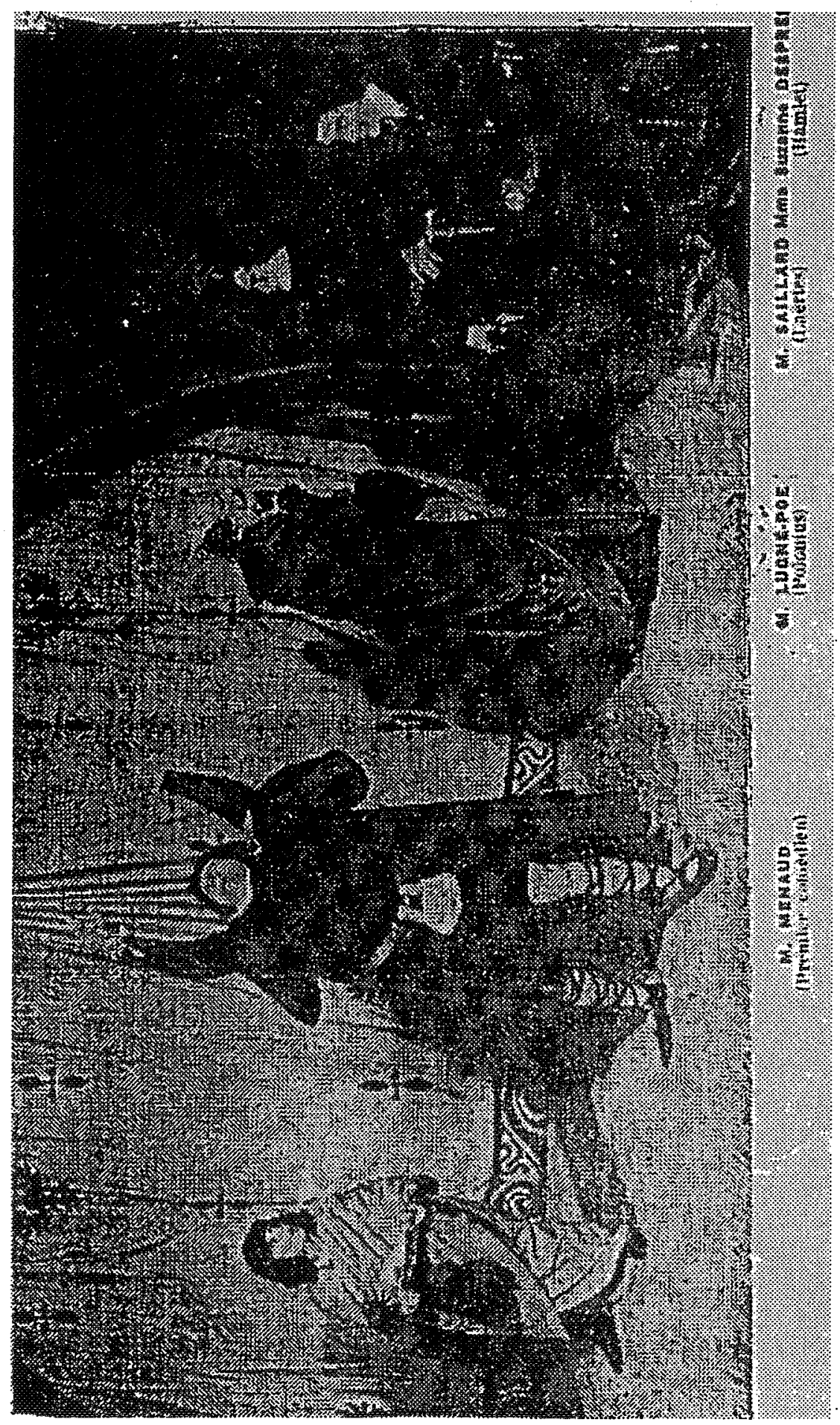

rllustration 6

Hamlet, Théâtre Antoine, 1913

Les comédiens 


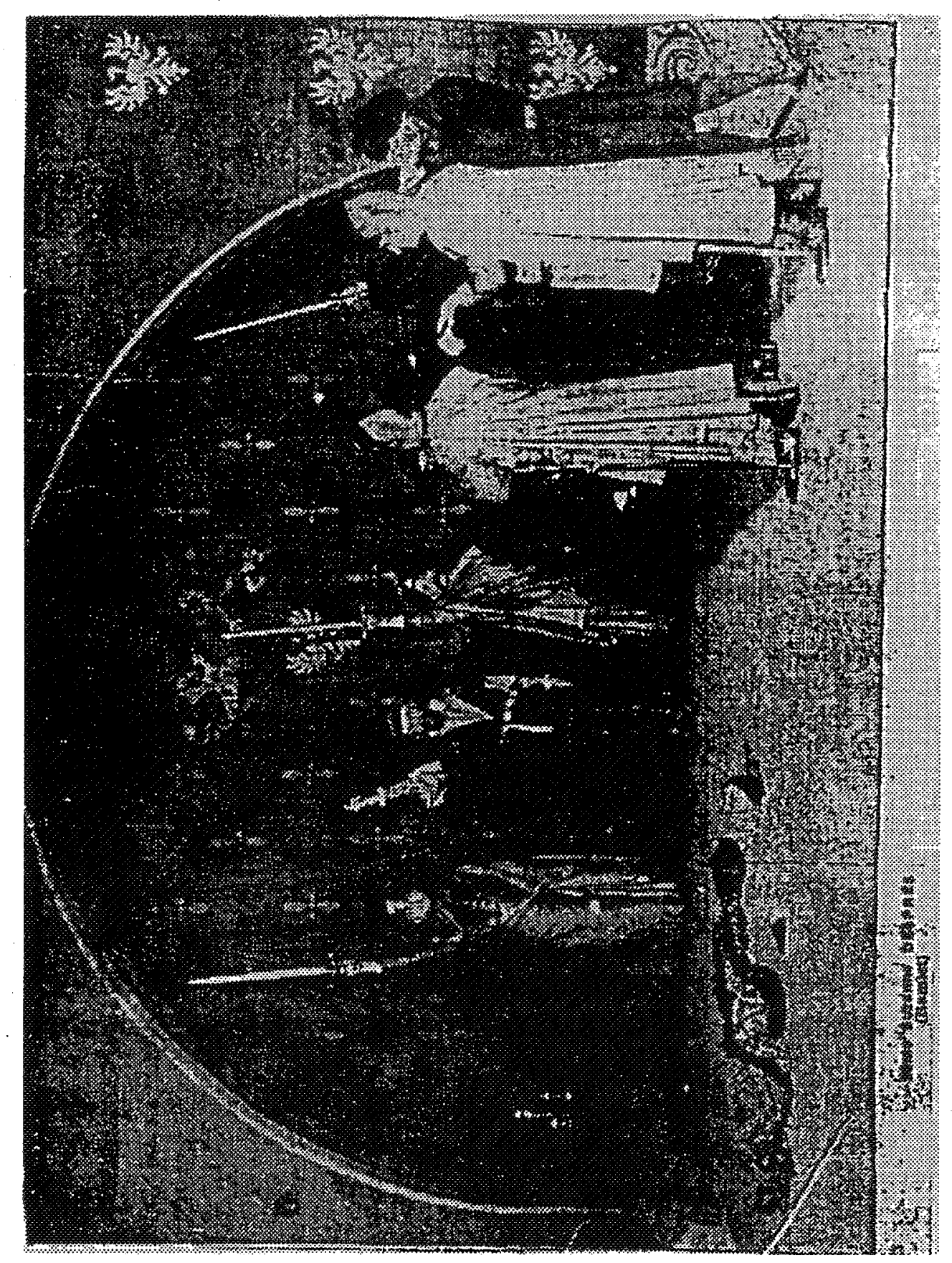

Illustration 7

Hamlet, Théâtre Antoine, 1913 Acte $V$, scène finale 
La scène est un espace vide, mis à part une touche de réalisme dans le traitement de la scène du cimetière : le premier fossoyeur, Firmin Gémier, creusait une véritable tombe, en rejetant des mottes de terre, mais celles-ci étaient jugées bien trop parcimonieuses pour la vraisemblance, et donc, cet espace tranchait fâcheusement avec la stylisation prédominante de la mise en scène.

Un seul objet scénique barre le centre de la scène dans certaines séquences : il s'agit d'un banc imposant qui ressort par la couleur claire de la pierre sur le fond sombre de la toile. cet unique élément à valeur polysémique, tantôt banc, tantôt autel médiéval de pierre, ou catafalque, amplifie le caractère linéaire du dispositif et du principe de mise en scène (illustration 4).

Lugné-Poe fit une utilisation très remarquée des lumières (il ne faut pas oublier qu'il fut l'un des premiers à équiper son théâtre de l'électricité, vers les années 1898, imposant une salle obscure pendant la durée du spectacle, au grand scandale des critiques de l'époque (la condamnation de Sarcey prête à sourire à présent), ne rallumant la salle que pour l'entracte. Son utilisation de lampes, qu'on ne peut pas encore appeler projecteurs, pour mettre en évidence un comédien, ou délimiter un espace naturellement clos, est une technique encore à ses balbutiements, qu'il finit par imposer par son évidence.

Le jeu est également simplifié et stylisé. Contrairement aux interprétations fameuses des périodes antérieures mais qui étaient encore dans les mémoires, Lugné-Poe refuse le principe du vedettariat d'un personnage, des numéros d'acteurs. Lugné-Poe et Suzanne Després avaient vu l'interprétation de Mounet-Sully à la Comédie Française en 1886 ainsi que celle de Sarah Bernhardt, d'abord Ophélie, sans cette même mise en scène et Hamlet, en 1899 dans son propre théâtre. Il s'agit là d'une conception «révolutionnaire»: cette mise en scène est conçue comme un travail de groupe qui doit frapper par sa cohérence interne, et ses effets du nombre, selon une logique longitudinale, comme on l'a déjà remarqué. Mais cependant, Suzanne Després dans le rôle principal d'Hamlet est très commentée. Ce n'est certainement pas la première fois qu'une femme prend le rôle sur la scène, les références et compilations abondent, en France, de «Madame Judith», sociétaire de la Comédie Française en 1866 à Sarah Bernhardt en $1899^{38}$. Les raisons invoquées habituellement comme inhérentes au caractère du personnage qui justifient le travestissement : il est hésitant, indécis, rêveur, n'ont pas de prise 
sur Suzanne Després. Par sa carrière antérieure, elle s'est distinguée comme interprète d'Ibsen et de Poil de Carotte (l'un de ses grands succès, aussi un rôle travesti), Suzanne Després compose un jeune homme qui prend substance sur la scène, qui impose sa présence par ses attitudes fermes et volontiers athlétiques, il manie l'épée et prend une posture conséquente qui s'impose face à Laertes, et surtout par l'intensité de son regard, les yeux exorbités grand ouverts, concentrés, tournés vers le dedans (illustration 5).

Une autre constatation s'impose : Hamlet frappe par son jeu de trois quarts. Gertrude, quant à elle, présente son profil dans un acte de soumission envers le discours d'Hamlet. On voit nettement le corps de Suzanne Després se détacher du fond de scène et aussi de la continuité de l'action. Il en est de même dans la scène avec les comédiens (illustration 6) : Hamlet est en dehors et en dedans tout à la fois. C'est également le principe de jeu de Polonius, souvent penché, presque de dos, qui lui aussi épie les autres, à la fois dans la cour et au-dehors pour observer, et cherche à percer un autre mystère.

Dans cette même scène on voit les comédiens se livrer à des envolées déclamatives emphatiques. C'est évidemment un jeu outré qui tranche avec les principes de diction qui caractérisent LugnéPoe et Suzanne Després. En effet, fidèles à leurs principes de jeunesse, ils pratiquent un phrasé très sobre qui se défend de l'exagération, un ton presque naturel, une diction qui bat en brèche les pratiques emphatiques.

Dans cette mise en scène, il n'y a pas de pénétration des acteurs dans la salle, comme dans Mesure pour mesure, toute l'action se passe sur la scène frontale. On a fait remarquer le placement de certains acteurs selon un principe de linéarité sous l'arche médiévale, face au public, empreints de cérémonial, puis le jeu particulier des deux investigateurs, Hamlet et Polonius. Je voudrais signaler un autre fonctionnement, rare à l'époque : à la séquence finale, les personnages au jardin se présentent dos aux spectateurs, selon une logique interne à la scène, c'est-à-dire tournés vers Fortinbras et ses acolytes, au fond, devenus le centre de focalisation (illustration 7). Cette gestuelle implique le principe du quatrième mur, comme si les spectateurs n'existaient pas, mais signifie aussi symboliquement une négation du libre arbitre des personnages et leur soumission à l'autorité nouvelle. Cette interprétation est renforcée par l'éclairage par le devant de la scène, si bien que les acteurs du premier plan perdent de leur consistance 
en même temps que leur identité, face aux arrivants, menaçants, dans la relative pénombre, leur épée brandie.

Comme je le disais, cette mise en scène a remporté un très grand succès. Des sommités comme le sculpteur Rodin, mais aussi William Poel en personne y ont assisté, une représentation supplémentaire a dû être organisée. C'était une reconnaissance de l'art singulier de Lugné-Poe, qui adoptait ici cependant une facture plus classique que dans Mesure pour mesure. Hamlet était auparavant considéré comme un drame individuel; dans sa conception à lui, et aussi grâce à sa participation comme acteur, la pièce devient la représentation d'une société tout entière, un microcosme. Il ouvrait la voie à une interprétation moderne.

\section{Conclusion}

Par rapport à la quantité invraisemblable de pièces qu'il a réussi à monter au cours de sa carrière (Lugné-Poe avait à une certaine époque jusqu'à huit mises en scène qui tournaient de par le monde), ces expériences shakespeariennes sont rares, mais très significatives. En effet, ces deux mises en scène, dans lesquelles il interprète un rôle qui fait appel à la verve et à la dissimulation permanentes, s'inscrivent à deux périodes clé de sa carrière théâtrale : la première, post- $U b u$, se situe dans ses débuts sulfureux, où il recherche le scandale par la nouveauté à tout prix, la deuxième, post-Annonce faite à Marie, représente une marche vers la maturité de son art. À cette époque-là, Lugné-Poe a acquis un nom sur la scène française et internationale. Mais il est intéressant de constater que ces deux tentatives n'ont pas lieu dans son Théâtre de l'Euvre, l'une parce qu'il voulait investir un espace renouvelé, l'autre, parce que ses moyens financiers sont momentanément réduits une fois encore. Donc, on pourrait parler de spectacles en marge de son activité ordinaire, dans des espaces d' "à côté».

Les principes qui régissent ces deux spectacles ne sont pas du tout semblables, mais cependant l'un et l'autre régis par des principes innovants, et qui s'inscrivent clairement dans sa conception d'un «Shakespeare sans décor» tel qu'il le définissait dans sa prise de position datant de 1897, à savoir une scène vide, libre, à l'élisabéthaine ou à l'italienne. Ce passage par Shakespeare ne s'est pas fait par le texte, comme il le pratiquait par ailleurs pour le répertoire scandinave ou français, mais directement par l'expérience théâtrale, à la recherche d'une «révolution» esthétique tout en revisitant la tradition, et en refusant fermement les critères 
de son époque. Mais on est obligé de constater que, même si certains metteurs en scène se sont inspirés de son art et de ses théories de façon oblique, il n'a fait ni école, ni disciple : jusqu'au bout, il a été une figure à part dans le théâtre français, un solitaire inspiré ou maudit, mais qui ouvre néanmoins au théâtre moderne.

Isabelle SCHW AR TZ-GASTINE

Université de Caen

\section{NOT E S}

${ }^{1}$ Jacques Robichez, Lugné-Poe, T.N.P., Collection «Le Théâtre et les Jours», Paris, L'Arche, 1955, p. 88.

2 Il l'inaugure en donnant Les Deux Veuves, de Félicien Mallefille, pièce dans laquelle il interprète le rôle du Garde-chasse.

${ }^{3}$ Dès ses débuts, il applique les consignes qu'il formulera plus tard, dans son article «À propos de "l'inutilité du théâtre au théâtre"», Mercure de France, oct-déc. 1896: «La diction ne doit pas sursauter au gré de la voix de l'acteur. C'est une harmonie parfaite, sans criaillerie. Au milieu de nous, l'acteur, pendant des scènes entières, s'efforcera de conter, de persuader, ses nerfs ne se révéleront que par l'humidité de ses yeux, l'angoisse de sa volonté qui imposeront le respect religieux...», p. 97.

${ }^{4}$ Voir les deux textes d'Émile Zola, Qu'est-ce que le naturalisme ? et Le naturalisme au théâtre.

${ }^{5}$ Il interprète le rôle de Goland.

${ }^{6}$ Voir l'article appréciatif d'Octave Mirbeau et aussi Fernand Nauzière, Lugné-Poe, Les célébrités de la scène française, Paris, Chiberre, 1921, p. 14.

7 1893: octobre: Rosmersholm, Ibsen, traduction de Prozor; novembre: Un Ennemi du Peuple, Ibsen, traduction de Chennevière et Johansen; décembre : Âmes Solitaires, Hauptmann, traduction A. Cohen. 1894: février: L'araignée de cristal, Rachilde, Au-dessus des forces humaines, Björnson, traduction de Prozor, Une nuit d'avril à Céos, de G. Trarieux, L'image, Maurice Beaubourg ; avril : Solness le constructeur, Ibsen, traduction de Prozor. Jacques Robichez, Lugné-Poe, op. cit., p. 198.

${ }^{8}$ Publié dans la revue L'Ermitage, septembre 1893, cité dans Jacques Robichez, Lugné-Poe, op. cit., p. 65. 
${ }^{9}$ Jacques Robichez, Lugné-Poe, op. cit. : «Euvre : un mot qui tout de suite a fait fortune. Il a rendu vigueur au verbe "œuvrer". Il a donné naissance à un néologisme et quelques poètes, acteurs, peintres se flattent encore aujourd'hui dans Paris, et dans le monde, d'avoir appartenu à cette société secrète des amis de Lugné, de ses collaborateurs de fondation ou de rencontre : les "Ouvriers"», p. 95.

10 Lors de sa rupture avec les Symbolistes, ceux-ci le traitent «d'entrepreneur de théâtre», Jacques Robichez, Le Symbolisme, ...

11 Il effectua une vingtaine de tournées à partir de l'année 1893-1894, jouant dans des villes ou même des villages où aucune troupe française n'était jamais allée, avec Eleonora Duse, Suzanne Després, Giovanni Grasso, Blanche Toutain, Isadora Duncan et tant d'autres. Il représentait le répertoire français, classique et contemporain, alors qu'il faisait connaître les œuvres scandinaves à Paris, mais aussi, donc, il faisait tourner des succès des ouvres autochtones. Ces tournées lui apportaient gloire théâtrale et finance.

12 «À propos de "l'inutilité du théâtre au théâtre"», Mercure de France, octobre-décembre 1896 , p. 90-8.

13 Jacques Robichez, Lugné-Poe, op. cit., p. 68 \& 120.

14. Maurice Denis est un ancien condisciple de Lugné-Poe au lycée Condorcet.

${ }^{15}$ Sous l'influence de leur ami Auguste Cazalis, ils avaient emprunté ce mot à l'hébreu : «nabi», au pluriel «nebiim», signifie «prophète».

«Une triple origine semble les réunir parfois: le préraphaélisme anglais, l'art du Japon, connu surtout par ses estampes, enfin l'engouement pour l'ornementation née de toutes les lignes végétales». Encyclopédie Universalis, volume 15, p. 1058-61.

Le peintre anglais Burne-Jones qui appartenait au mouvement préraphaélite, a également collaboré aux décors de l'Euvre. Encyclopédie Universalis, volume 15, p. 1058-61.

${ }^{16}$ Ces artistes nabis ont également collaboré au Théâtre d'Art fondé en 1890 par Paul Fort, alors âgé de 18 ans, qui voulait se poser en rival d'André Antoine et de son Théâtre-Libre régi selon les principes de l'école naturaliste.

17 Nozière, op. cit., p. 7.

18 Voir par exemple Jacques Robichez, Le Symbolisme au Théâtre, Lugné-Poe et les débuts de l'Euvre, Paris, L'Arche, 1957 et Gertrude $\mathrm{R}$. Jasper, Adventure in the Theatre, Lugné-Poe and the Thêâtre de l'QEuvre to 1899, New Brunswick, Rutgers University Press, 1947, p. 222-36.

19 Lugné-Poe, La Parade, volume 2, Acrobaties, souvenirs et impressions de Théâtre 1894-1902, NRF, Paris, Gallimard, 1931, p. 243.

${ }^{20}$ D'après Suzanne Després, il ne parlait pas anglais, mais il réussissait toujours à se faire comprendre partout où il passait par un mimétisme 
linguistique doublé d'une verve éclairante; il aurait, dit-on, traduit cette pièce avec l'aide de son père (voir Jacques Robichez). 1954.

${ }^{21}$ Robert Speaight, William Poel and the Elizabethan Revival, Londres,

${ }^{2}$ Ibid.

${ }^{23}$ Lugné-Poe, «Shakespeare sans décors, la société Élisabéthaine à Londres», dédié à Octave Mirbeau, La Nouvelle Revue, mars-avril 1897, p. 143-52.

${ }^{24}$ Il est possible qu'il ait également assisté aux spectacles précédents : 1895: La Nuit des rois, La Comédie des erreurs, 1896: Mesure pour mesure, Le Docteur Faust.

25 Lugné-Poe, «À propos...».

26 Jacques Robichez, Le Symbolisme au théâtre, Lugné-Poe et les débuts de l'œuvre, Paris, L'Arche, 1957, p. 306. Il cite Burlington Hall, Gray's Inn Hall, St George's Hall, Merchant Taylors' Hall qui ont donné «asile» au metteur en scène.

${ }^{27}$ Lugné-Poe, «Shakespeare sans décors», p. 149-50: «by kind permission of the court of the Company». «La Société Élisabéthaine pousse le scrupule un peu naïf jusqu'à s'efforcer de jouer dans le lieu même, ou du moins sur l'emplacement de l'endroit où ont été créées les pièces. Je sais que, cet hiver, il en sera ainsi pour Mesure pour mesure, qui sera représentée dans le hall du Middle Temple, avec la permission des banquiers, précisément à l'endroit où Shakespeare joua la pièce il y a trois siècles».

${ }^{28}$ Lugné-Poe, «Shakespeare sans décors», op. cit., p. 151.

29 «[O]n était parvenu à restituer le texte exact, en sorte que les mots pouvaient être admis pour canoniques et que le respect avait été si bien établi qu'il était impossible d'intercaler les paroles de tout autre écrivain que le maître». Lugné-Poe, «À propos de "l'inutilité du théâtre au théâtre"”, Mercure de France, oct-déc. 1896, p. 90-8.

${ }^{30}$ Lugné-Poe, Acrobaties, op. cit., p. 244. Le verbe «révolutionner» est répété deux fois sur cette même page, indiquant bien le but ultime poursuivi par le metteur en scène.

31 Jacques Robichez, Lugné-Poe: «Roméo, Timon d'Athènes, Une tragédie dans l'Yorkshire», p. 84.

${ }_{32}$ Lugné-Poe, «À propos de "l'inutilité du théâtre au théâtre"», Mercure de France, oct-déc. 1896, p. 90-8.

33 «Le proscenium, très vaste, entre, pour ainsi dire, dans la salle, sans rampe, sans séparation, ce qui est très nuisible à l'illusion», écrit Henri Fouquier. Voir le Micro-film du fonds Rondel, p. 15.

${ }_{34}^{34}$ Lugné-Poe, Acrobaties, op. cit., p. 244-245.

35 Jacques Robichez indique que plusieurs écrivains portaient alors ce nom, ce qui n'est pas sans poser de problème de différenciation. La Revue 
Blanche du 1er juillet 1896 publie un article de Louis Ménard, se plaignant d'avoir été plagié par M. Meurice dans sa traduction en vers de Hamlet, p. 19-26. Louis Ménard aurait également traduit Othello.

${ }^{36}$ Lugné-Poe, Acrobaties, op. cit., p. 244.

${ }^{37}$ Lucien Muhlfeld, Les Échos, 13.12.1898.

${ }^{38}$ Dossier de l'Arsenal, revue de presse. 